\title{
Recognizing failures in citizen science projects: Lessons learned
}

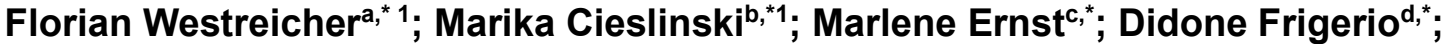 Barbara Heinische, ${ }^{\mathrm{e},}$; Thomas Hübner ${ }^{\mathrm{f},{ }^{*}}$ \& Johannes Rüdisserg, ${ }^{,}$}

a Office of Public Relations, University of Innsbruck

Innrain 52, 6020 Innsbruck, Austria

E-mail: florian.westreicher@uibk.ac.at

b OeAD-Center for Citizen Science

Universitätsstraße 5, 1010 Vienna, Austria

E-mail: marika.cieslinski@oead.at

c Science \& Technology Hub, University of Salzburg Jakob-Haringer-Str. 6/Techno 6, 5020 Salzburg, Austria E-mail: marlene.ernst@sbg.ac.at

d Konrad Lorenz Research Center, Core Facility for Behaviour and Cognition \& Department of Behavioural and Cognitive Biology, University of Vienna

Fischerau 11, 4645 Grünau im Almtal, Austria

E-mail: didone.frigerio@univie.ac.at

e Centre for Translation Studies, University of Vienna

Gymnasiumstraße 50, 1190 Vienna, Austria

E-mail: barbara.heinisch@univie.ac.at

\section{$f$ Zentralanstalt für Meteorologie und Geodynamik}

Section Climatology

Division Customer Service

Hohe Warte 38, 1190 Vienna, Austria

E-mail: thomas.huebner@zamg.ac.at

$g$ Department of Ecology, University of Innsbruck

Sternwartestraße 15, 6020 Innsbruck, Austria

E-mail: johannes.ruedisser@uibk.ac.at

* All authors contributed equally to the manuscript 
Failures are an immanent part of academic processes driving research progress. Therefore, they are also present in citizen science projects. Still the research community tends to highlight the successes while willingly omitting the mistakes and thus, withholding important information from their peers. This article presents results from an interactive conference workshop, during which participants discussed their experience of failures in citizen science projects and proposed anti-fail tips to enhance processes in the future. With the workshop and this article the authors want to contribute to the establishment of an error culture in citizen science.

Austrian Citizen Science Conference 2020 - ACSC2020

14-16 September 2020

Vienna - Austria 


\section{Introduction}

In a conversation about a scientific - or specifically a citizen science - project a point will be reached where someone is going to ask, "How did you manage all this so well?". Generally, conference talks and personal dialogues underline the very best of all outcomes whilst too often omitting struggles and mistakes. Nevertheless, these are relevant steps for individual learning as well as for the potential improvement in other projects. Failures in the scientific process may bear a lot of information [1,2] but are rarely communicated in scientific publications or conference talks $[3,4]$. Sharing failure experiences within the community empowers progress in research.

In the present contribution, we address the topic of failures in citizen science projects by discussing the outcomes of a 'failure session', which was conducted at the Austrian Citizen Science Conference 2020. For this purpose, a multidisciplinary team was gathered and an online survey on failures in citizen science projects was launched. Thirty-eight anonymous scientists joined the survey, contributing additional evidence, which was considered when designing the workshop. Further failure experiences were investigated and shared among the workshop participants.

\section{Methods}

Our initial plan of conducting an on-site workshop was changed due to the COVID-19 precautions and new conference modalities. Therefore, we designed a workshop with the online conference platform and a digital whiteboard tool. Interactivity was a main objective as the aim of the workshop was to encourage attendees to share their failures, thereby benefitting from each other's experiences. The participants came from various research institutions and disciplines, which contributed to a better overall picture of failures in citizen science projects. However, the majority of the attendants belonged to the natural sciences or the humanities.

The workshop consisted of three consecutive parts. In the beginning, we invited participants to answer the following questions on a digital whiteboard a) "In which failure categories did failures happen?" (e.g. methods, communication, publication) b) "In which project phase did you experience failures?". We differentiated between the phases project planning (including gathering the idea/research design), execution (data collection and data analysis) and dissemination. The participants' fields of research were identified by using different color codes. The following domains were available: humanities, engineering and technology, natural sciences, social and economic sciences, arts, other. Afterwards, the responses were complemented with case scenarios from different research disciplines presented by the five authors. For the last part and wrap-up of the failure workshop, we returned to the digital whiteboard. Participants were encouraged to suggest 'anti-fail tips' indicating what measures should be implemented on a meta-level which might serve to promote a culture fostering the communication about failures. Attendants were again able to cluster their answers according to their academic disciplines. 


\section{Results}

\subsection{Failure categories}

We identified eight fields for possible failures in a project (Figure 1). In total, 21 participants from four different fields of knowledge reported 34 fails. The majority of which were assigned to the categories communication (32\%) and complexity of the tasks (23\%). However, also the categories time management $(18 \%)$ and methodology (15\%) seem to be failure-sensitive, especially among researchers from the natural sciences. One failure each was mentioned for the categories formulating research hypothesis and funding and none were reported for the publication/dissemination category.

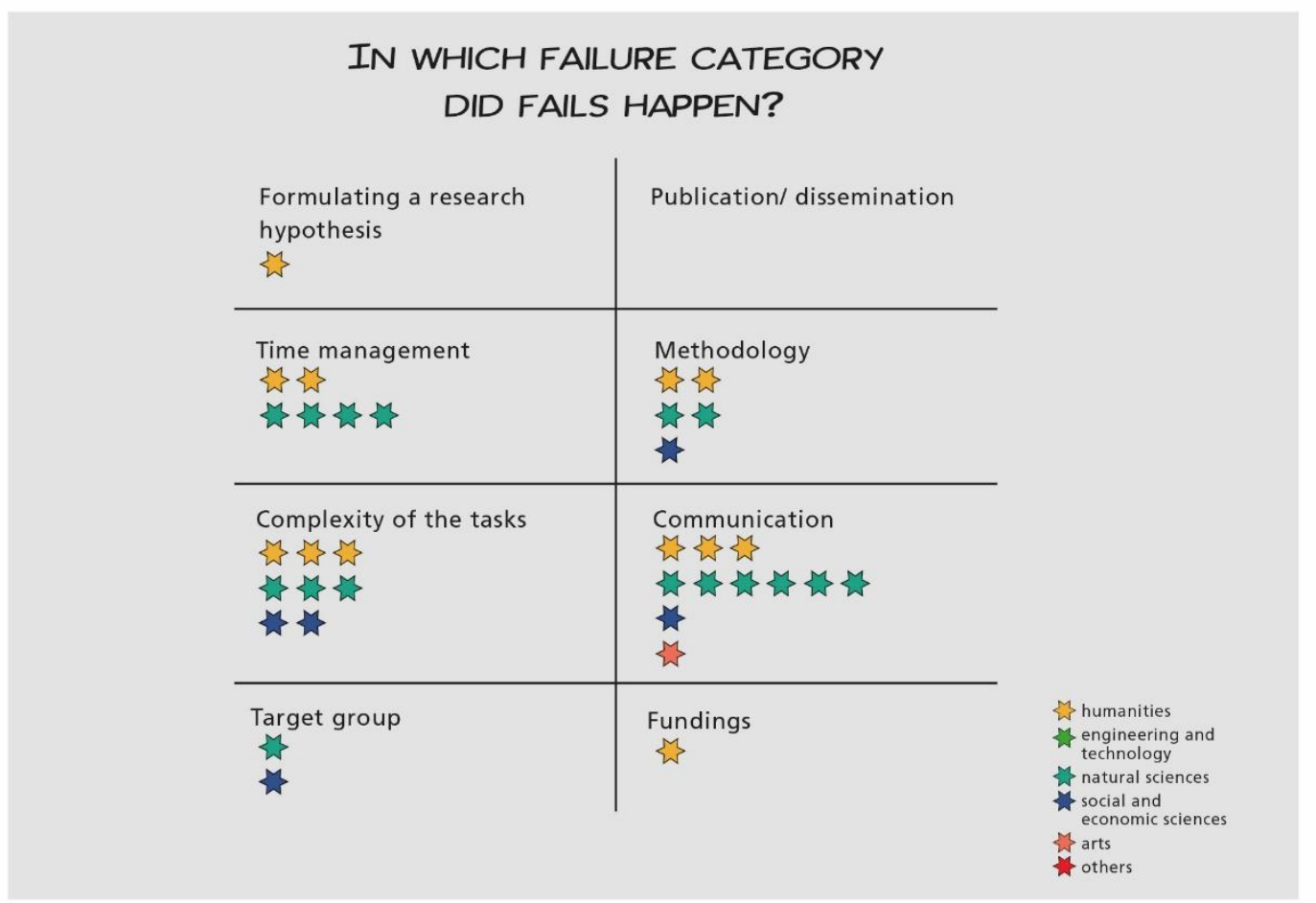

Figure 1 - Failure categories reported in citizen science projects from different fields of knowledge

\subsection{Failures related to project phases}

In part b) of the workshop, additional information was given concerning fails in specific project phases (Figure 2): The execution phase (i.e. data collection and analysis) had the highest potential for failure as many problems were reported when citizen scientists were working with data and tools. Technical and digital aspects should not be underestimated, e.g. the technology did not work, or the citizen scientists could not handle the tools as expected. These digital hurdles were reported by citizen science projects in the humanities, engineering/technology, and natural sciences.

The project phases planning, execution and dissemination also had the potential to be more timeconsuming than initially anticipated. Communication beforehand (in order to reach potential citizen scientists), communication during project implementation and the dissemination of results 
were mentioned as key elements of successful projects, which are particularly time-intensive when working with many participants. Especially the execution phase was fraught with timeconsuming hurdles, as well.

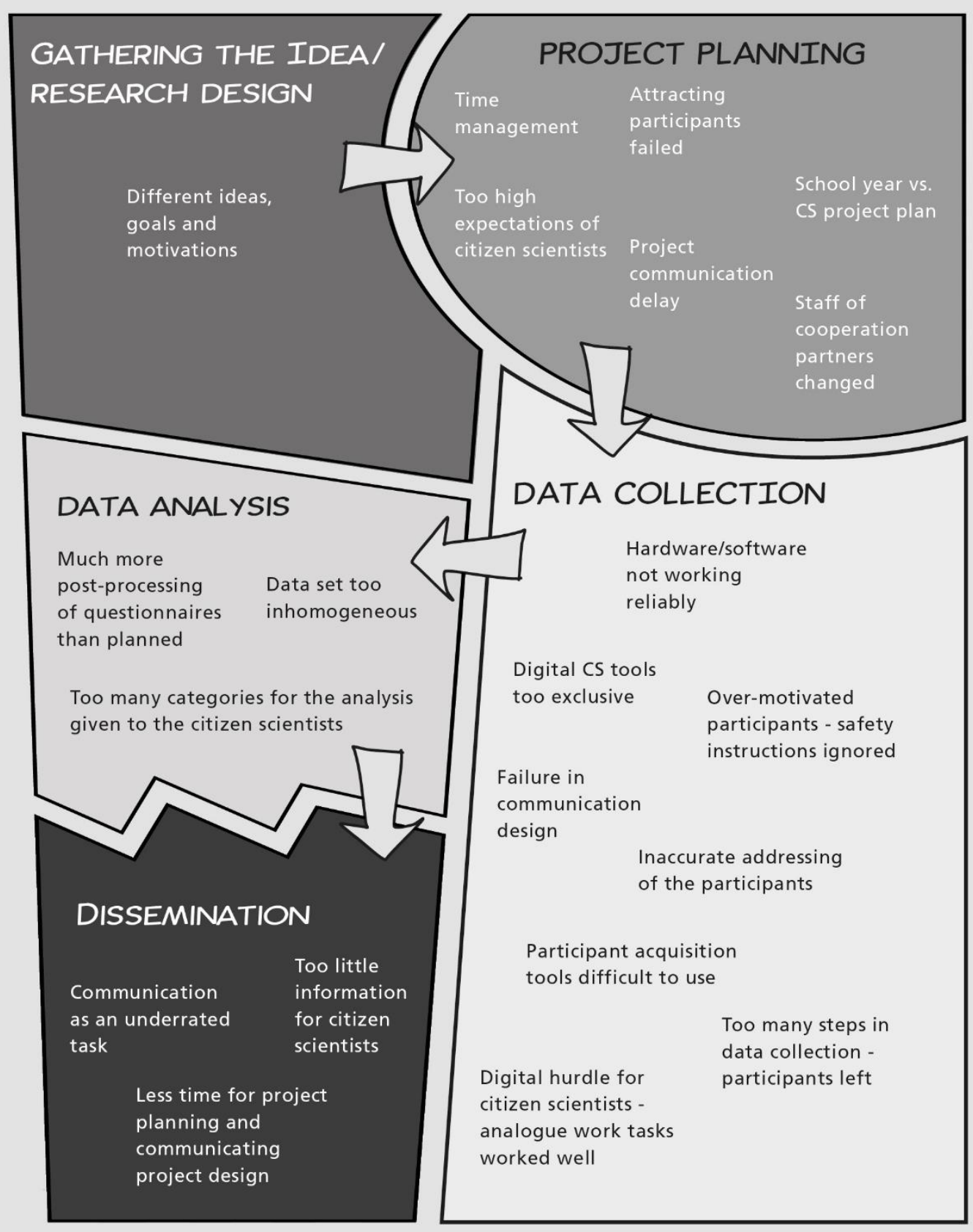

Figure 2 -Failures related to the different phases in a citizen science project

\subsection{Lessons learned and recommendations at a meta-level}

The 'anti-fail tips' collected during part c) of the workshop addressed various aspects, which may contribute to making the preparation, execution and dissemination of citizen science projects more efficient (Figure 3). Regardless of the discipline, researchers may have to adapt their project design continuously throughout the process. It was recommended to clarify expectations in advance and 
get to know your target group, including their availability. Clear instructions, guidelines and rules, e.g. for data collection, e.g. predefined answers that participants can tick and avoiding open question formats were tips provided. Additionally, citizen science requires flexibility since not everything will work out as initially planned. Another important factor is communication (at eye level) throughout the project. Constant interaction is required to keep the motivation high and feedback helps to improve data quality. Moreover, it is crucial to keep different communication cultures, working styles and objectives in mind. It was also recommended that citizen science project coordinators consider ('unusual') cooperation partners, e.g. schools. 

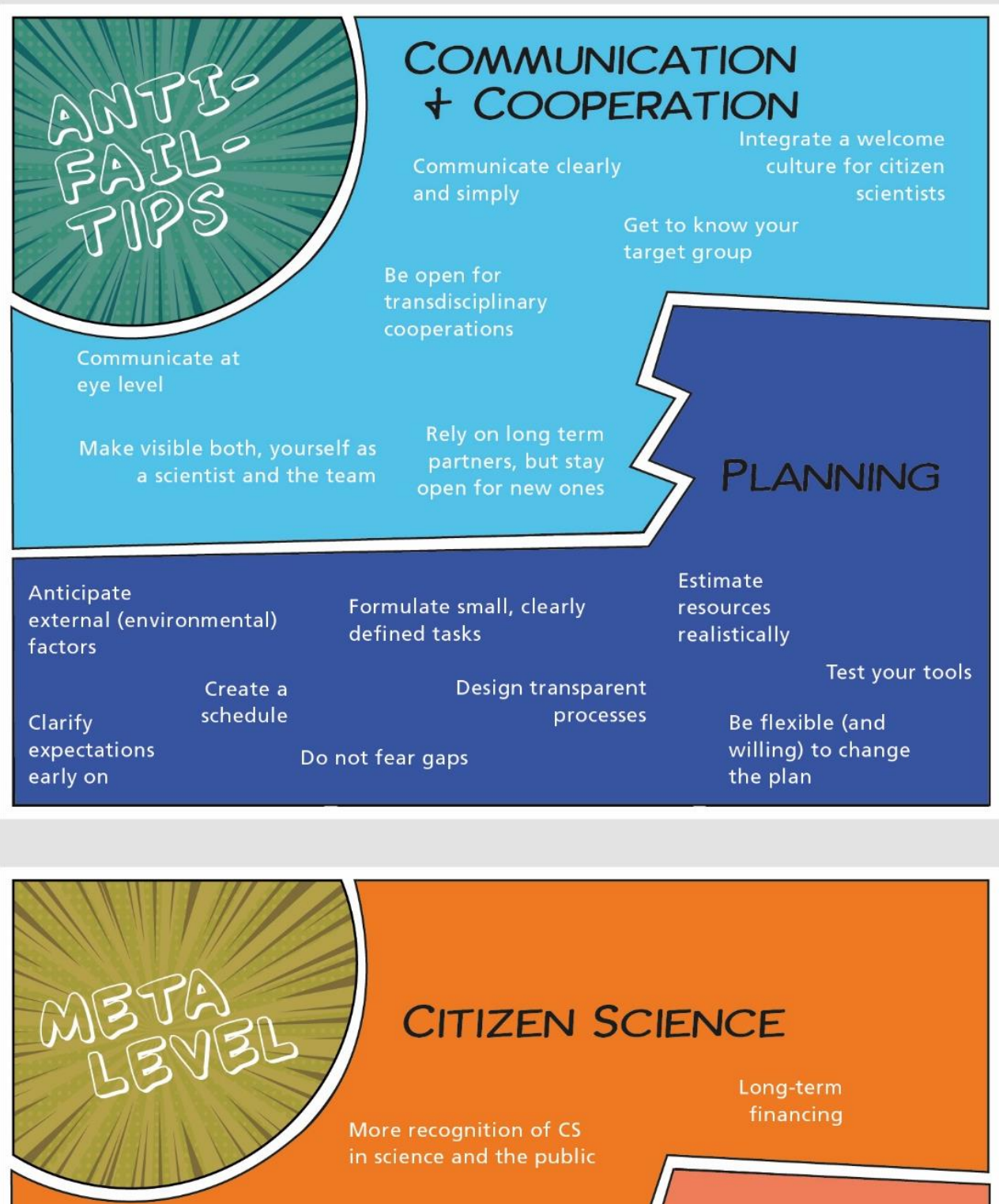

\section{CITIZEN SCIENCE}

More recognition of CS in science and the public

CS in teacher training and PhD curricula

More science about CS

Collection of

fails - Share your

fails proudly!

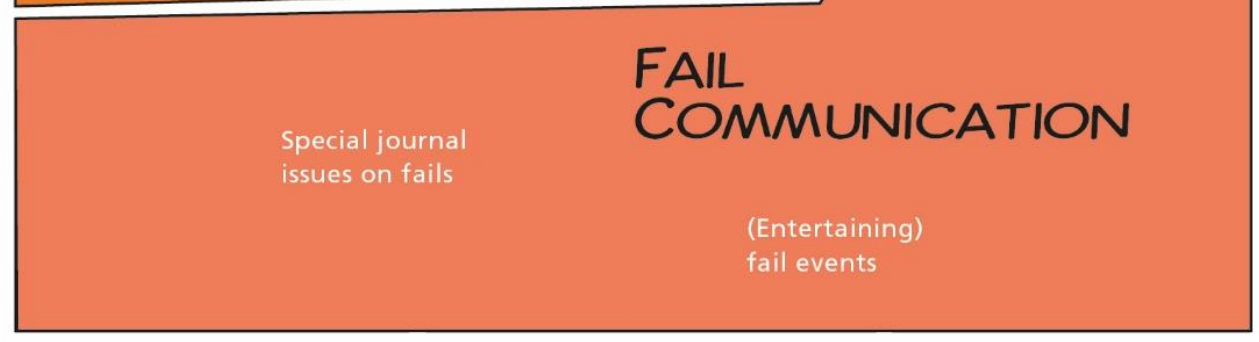

Figure 3 -Anti-fail tips and synopsis at a meta-level 


\section{Discussion}

Failure is part of the academic process and a driver for its advancement [5]. A well-designed experiment starts with the objective to falsify the null hypothesis which nevertheless very often turns out to be true. Basically, science is successful because of failures and not despite them [6]. Failures frequently promote a basic objective of science: discovery. This applies also to citizen science, an old and at the same time modern and recently rising approach to research. The fast development and expansion of citizen science enables and demands the proactive elaboration of a positive approach to deal with mistakes and errors. Disciplines that manage to establish an error culture combined with systematic failure analysis (e.g. aviation or mountaineering) enable a highly dynamic development in their respective fields.

Due to their inter- and often transdisciplinary approaches citizen science projects are frequently highly complex and demanding endeavours. Hence, it is comprehensible that $55 \%$ of the reported failures were related to communication and the complexity of the tasks. Both digitally supported and in-person communication as well as complex management are the backbone of various citizen science projects. This is in line with many of the recommendations ('anti-fail tips') collected during the workshop. These focus on the importance of a "stringent, clear, straightforward communication at eye level" and an "early, transparent, and realistic planning" considering "available resources", which allows to react to "uncontrollable external factors" and a "flexible adaptation of the plan", if needed.

In order to establish the aforementioned error culture in citizen science and to promote mutual learning from existing and planned projects, it is crucial to talk not only about open data but also about open failure, hence actively promoting the exchange of experience that includes mistakes. This could be part of conferences, science communication events or publications. Finally, understanding errors and their importance for academic success, contributes to a better understanding of the academic process per se.

One of the key findings is that talking about failures and having the chance to speak about them can be very enlightening and helpful for individual academic careers and a guideline for other projects in order to avoid repeating the same failures. Thus, implementing failure workshops such as this one could be a first step towards co-created processes [7] and help amplify the citizen science community. A redefinition of failures into lessons learned may be a vital first step to accept them as a crucial part of development and discovery. In the words of a famous British author: "Failure taught me things about myself that I could have learned no other way" [8].

\section{Acknowledgement}

We thank all workshop participants for their valuable contributions and active collaboration as well as the 38 anonymous colleagues that were responding to the online survey before. Thanks to the organizers of the Austrian Citizen Science Conference 2020 for the opportunity to conduct the workshop. We also thank Catharina Walli from the University of Innsbruck, Office of Public Relations, for her patience in creating the illustrations.

\section{References}

[1] R. Rosenthal, The file drawer problem and tolerance for null results, Psychological Bulletin, 86 (1979) 3 [DOI: 10.1037/0033-2909.86.3.638]. 
[2] D. Asingizwe, P.M. Poortvliet, C.J.M Koenraadt, A.J.H.van Vliet, C.M. Ingabire, L. Mutesa, et al., Why (not) participate in citizen science? Motivational factors and barriers to participate in a citizen science program for malaria control in Rwanda, PLoS ONE 15 (2020) 8 [DOI: 10.1371/journal.pone.0237396].

[3] C.G. Druschke, C.E. Seltzer, Failures of engagement: lessons learned from a citizen science pilot study, Appl Environ Educ Commun 11 (2012) 3-4 [DOI: 10.1080/1533015X.2012.777224].

[4] K. Arts, Y. Melero, G. Webster, N. Sharma, N. Tintarev, E. Tait, C. Mellish, S. Sripada, A. MacMaster, H. Sutherland, C. Horrill, X. Lambin, R. van der Wal, On the merits and pitfalls of introducing a digital platform to aid conservation management: Volunteer data submission and the mediating role of volunteer coordinators, J Environ Manage 265 (2020) [DOI: 10.1016/j.jenvman.2020.110497].

[5] J. Loscalzo, A Celebration of Failure, Circulation 129 (2014) 9 [DOI: 10.1161/CIRCULATIONAHA.114.009220].

[6] A. Barwich, The Value of Failure in Science: The Story of Grandmother Cells in Neuroscience, Front Neurosci 13 (2019) [DOI: 10.3389/fnins.2019.01121].

[7] F. Heigl, B. Kieslinger, K.T. Paul, J. Uhlik, D. Frigerio, D. Dörler, Co-Creating and Implementing Quality Criteria for Citizen Science, Citizen Science: Theory and Practice 5 (2020) 1 [DOI: http://doi.org/10.5334/cstp.294].

[8] J.K. Rowling, Very Good Lives. The Fringe Benefits of Failure and the Importance of Imagination, Sphere, London 2015. 